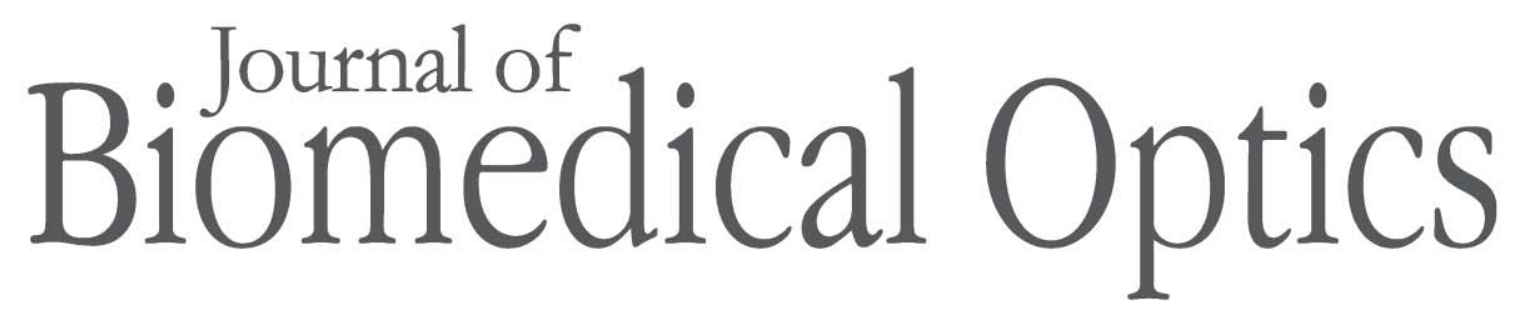

SPIEDigitalLibrary.org/jbo

\title{
Fiber-based combined optical coherence and multiphoton endomicroscopy
}

Gangjun Liu

Zhongping Chen 


\title{
Fiber-based combined optical coherence and multiphoton endomicroscopy
}

\author{
Gangjun Liu, ${ }^{\mathrm{a}, \mathrm{b}}$ and Zhongping Chen ${ }^{\mathrm{a}, \mathrm{b}}$ \\ a University of California, Irvine, Beckman Laser Institute, Irvine, California 92612 \\ bUniversity of California, Irvine, Department of Biomedical Engineering, Irvine, California 92697
}

\begin{abstract}
A fiber-based multimodal imaging system that combines multiphoton microscopy (MPM) with Fourier domain optical coherence microscopy (OCM) is reported. The system uses a fiber-based femtosecond laser, a fiber coupler, and a double-clad fiber (DCF) device. The fiber laser has a central wavelength of $1.04 \mu \mathrm{m}$ and bandwidth of $29 \mathrm{~nm}$. Longer excitation wavelength is used to increase penetration depth and increase the excitation efficiency for dyes, such as red fluorescent dyes. A single mode fiber coupler is used to replace the free-space beam splitter and one arm of the coupler is fused with a double-clad fiber device. The MPM and OCM share the same excitation light path in the core of a double-clad fiber, while the OCM and MPM signals were collected by the core and clad of the double-clad fiber, respectively. The performance of the introduced double-clad device is analyzed. The device can confine all the excitation light in the core and has a collection efficiency of $20 \%$ for the MPM signal. The efficiency can be further increased by fusing more multimode fibers with the DCF. Simultaneous optical coherence microscopic imaging, second harmonic generation imaging, and two-photon excitation fluorescence imaging are demonstrated in biological samples. @ 2011 Society of Photo-Optical Instrumentation Engineers (SPIE). [DOI: 10.1117/1.3555180]
\end{abstract}

Keywords: optical coherence microscopy; multiphoton microscopy; multimodal imaging; scanning microscopy; fiber device.

Paper 10669LR received Dec. 21, 2010; revised manuscript received Jan. 21, 2011; accepted for publication Jan. 23, 2011; published online Mar. 29, 2011.

There has been great interest in combining nonlinear optical contrast multiphoton microscopy (MPM) with linear contrast optical coherence microscopy (OCM). In OCM, imaging contrast originates from the inhomogeneities of sample scattering properties that are dependent on sample refractive indices. In many instances, changes in sample linear scattering properties are small and difficult to measure. MPM, on the other hand, is based on multiphoton excited fluorescence and/or harmonic generation and provides molecular contrast and specificity. Combining nonlinear optical contrast with OCM will greatly enhance clinical applications of these imaging modalities. A number of groups, including ours, have demonstrated this multimodality imaging in a benchtop microscopy with Ti:Sapphire laser. ${ }^{1-5}$ However, all of the reported systems are implemented in a benchtop free space microscopy configuration, which limits their potential applications for in vivo animal and clinical imaging. Fiber-based systems have been widely used in OCT systems, and fiber-based MPM systems have also been reported by several groups. ${ }^{6,7}$ A fiber-based combined OCM/MPM system has not been demonstrated. A fiber-based system reduces system complexity and minimizes the requirement of optical alignment. Integration of a femtosecond fiber laser as the source for combined OCT/MPM provides more flexibility and lower cost. Recently, MPM with a longer excitation wavelength than the traditional $800 \mathrm{~nm}$ range has been shown to increase the penetration depth. ${ }^{8}$ In addition, MPM with a longer wavelength source beyond $1 \mu \mathrm{m}$ could increase the excitation

Address all correspondence to: Zhongping Chen, University of California, Irvine, Beckman Laser Institute, Irvine, California 92617. Tel: 949-824-1247; Fax: 949824-8413; E-mail: z2chen@uci.edu. efficiency for dyes such as red fluorescent dyes, and dispersion at $1 \mu \mathrm{m}$ is less than that at $800 \mathrm{~nm}$.

In this letter, we demonstrate a fiber-based combined Fourier domain OCM/MPM system with a single fiber laser source, a fiber coupler, and a double-clad fiber device. The laser source has a central wavelength of $1.04 \mu \mathrm{m}$, and a bandwidth of $29 \mathrm{~nm}$. The system used a $2 \times 2$ fiber coupler and specifically, a double-clad fiber-(DCF)-based multimode device: a $(2+1): 1$ pump/signal combiner (PSC) was introduced to enhance the MPM signal collection and separate the OCM and MPM signal. The performance and benefits of the $(2+1): 1$ PSC device were analyzed for the combined OCM/MPM applications. Multimodal images of biological samples were demonstrated.

The combined OCM/MPM system is schematically illustrated in Fig. 1(a). A fiber-based femtoscecond laser (FBFL) was used as the light source for both OCM and MPM. The detail of the FBFL can be found in Ref. 7. Briefly, the FBFL delivered a sub-100, fs pulse with average power of more than $1 \mathrm{~W}$. The repetition rate was $76 \mathrm{MHz}$. The output spectrum is shown in Fig. 1(b). The FWHM spectrum bandwidth is $29 \mathrm{~nm}$, which gives an axial resolution of $16.1 \mu \mathrm{m}$ in air for the OCM.

The laser output is sent to a grating pair dispersioncompensation stage and then focused with a $20 \times$ objective, into one arm of a single mode (SM) $2 \times 2$ fiber coupler. The coupling efficiency is $30 \%$. The coupled laser power is equally split by the coupler into the sample and reference arm. In the sample arm, the SM fiber of the coupler was fused with one end of a DCF based PSC. Another end of the PSC was angle cleaved to reduce the reflection. The DCF of the PSC was terminated with a subminiature version A (SMA) connector and connected with a handheld

1083-3668/2011/16(3)/036010/4/\$25.00 @ 2011 SPIE 


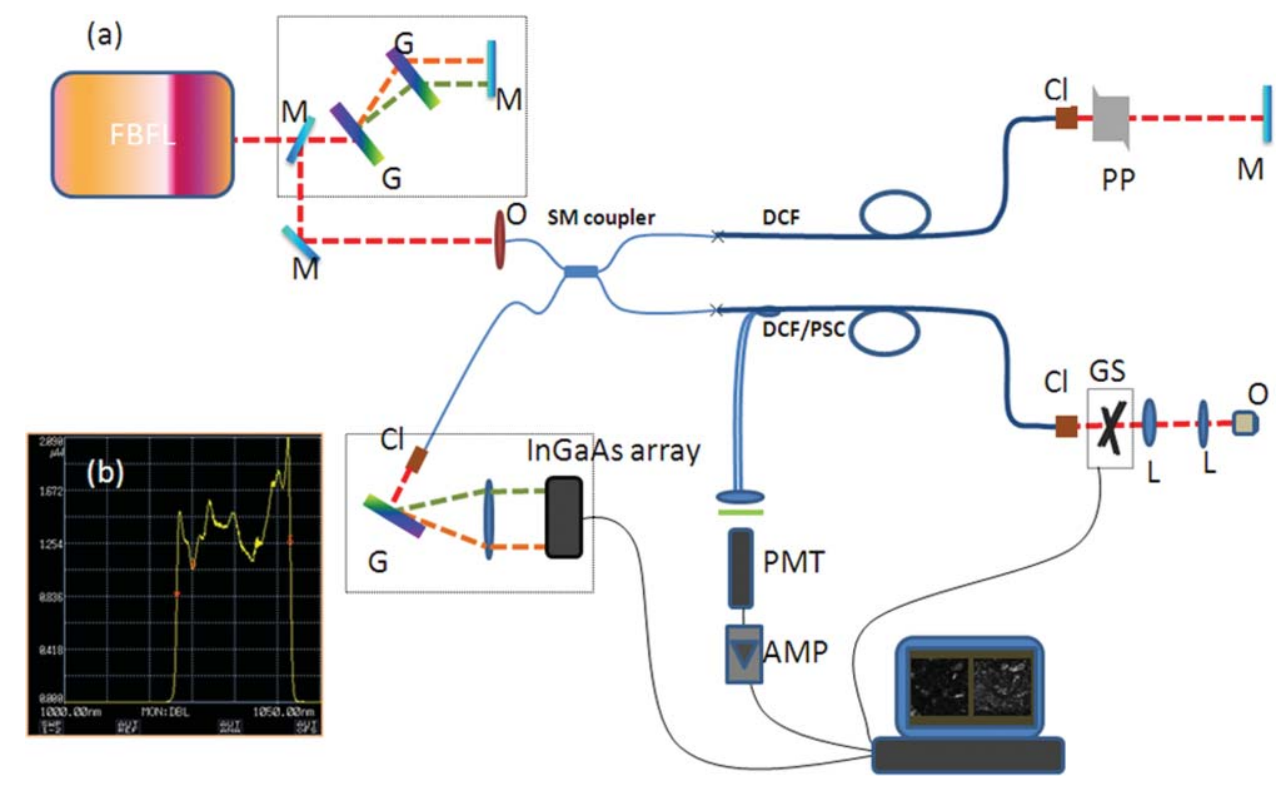

Fig. 1 (a) Scheme of the system setup. FBFL: fiber based femtosecond laser; M: mirror; G: grating; O: objective; SM coupler: single mode coupler; DCF: double-clad fiber; PSC: pump/signal combiner; CL: collimator; PP: prism pair; GS: galvo mirror scanner; L: lens; PMT: photomultiplier tube; AMP: low noise pre-amplifier. (b) Spectrum of the fiber laser.

probe. The handheld probe includes two axial galvo mirror scanners, a scanning lens, a relay lens, and an objective. The pulse width at the sample side is $300 \mathrm{fs}$ after dispersion compensation. In the reference arm, a double-clad fiber with core size of 10 $\mu \mathrm{m}$ was spliced to the SM fiber of the coupler in order to match the property (dispersion, nonlinear effect, etc.) of the reference arm. The other side of the DC fiber was angle cleaved to reduce the backreflection. A prism pair was used to match the dispersion induced by lenses and objective in the sample arm. The interference signal between the reference arm and sample backscattering or backreflection signal was sent to a homebuilt spectrometer. The home-built spectrometer included a grating with 1200 grooves $/ \mathrm{mm}$, an achromatic lens with $300 \mathrm{~mm}$ focusing length and an InGaAs detection array (SU1024-1.7T, Sensors Unlimited, Princeton, New Jersey). The InGaAs detection array has 1024 pixels with a pixel size of $25 \times 25 \mu \mathrm{m}^{2}$ and a maximum line speed of $7.7 \mathrm{KHz}$. Compared with the time domain OCM system, the Fourier domain OCM system has benefits of a better signal/noise ratio, faster speed, and nonmoving components. In addition, with the Fourier domain technique, the entire depth information can be obtained at once without the necessarily of depth scanning. The OCM data processing includes spectrum calibration, fast Fourier transformation (FFT), and logarithmic expression of the signal amplitude obtained by FFT. A linear interpolation was used to transform the spectrum from linear wavelength array to linear optical frequency array.

Double-clad fibers have been widely used in endoscopic and handheld MPM systems to increase the MPM signal collection efficiency. ${ }^{7,9}$ For more compact systems, single mode fiber couplers, double-clad fiber couplers, and double-clad photonic crystal fiber couplers have also been investigated. ${ }^{6,10,11}$ In this letter, we introduce a PSC for the purpose of delivering the OCM and MPM excitation light and collection of the OCM and MPM signal. The pump/signal combiner has been widely used in the field of high power fiber lasers to couple one or several high power pump lights into one DCF. ${ }^{12}$ A $(2+1): 1$ pump/signal combiner is schematically shown in Fig. 2 . A $(2+1): 1$ pump/signal combiner includes two multimode fibers (ports 2 and 3 in Fig. 2) fused with one DCF. The core diameter and refraction index of the multimode fiber are matched with the diameter and refraction index of the inner clad of the DCF so that fusion loss is minimal. The DCF used in this experiment has a core diameter size of $10 \mu \mathrm{m}$ and inner-clad diameter of $105 \mu \mathrm{m}$. The numerical aperture for the core and inner-clad of the DCF is, respectively, 0.05 and 0.45 . The high NA of the inner-clad helps to increase the signal collection efficiency, especially for the weak MPM signal. In the experiment setup, port 1 of the combiner was fused with the sample arm of the fiber coupler. The fiber in the coupler has a core diameter of around $6 \mu \mathrm{m}$ which is different from the $10 \mu \mathrm{m}$ core diameter of DCF in the PSC. The splice loss between the two fibers is estimated to be around $1 \mathrm{~dB}$. Because the excitation light is first focused into a SM fiber and then guided into the core of the DCF fiber, all of the excitation light can be confined in the core of the DCF.

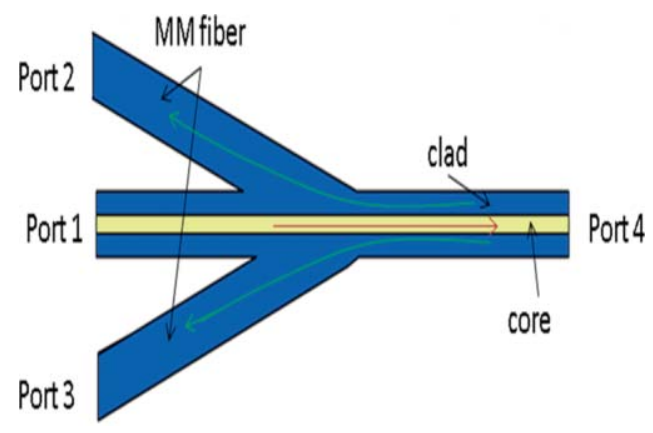

Fig. 2 Scheme of a $(2+1): 1$ pump/signal combiner. The right-pointing arrow indicates the excitation light path and the left-pointing arrow indicates the MPM signal collection path. 
The backscattered excitation light will be collected by both the core and the inner-clad of the DCF through port 4 (Fig. 2). However, only the backscattered excitation light in the core is finally sent to interfere with the OCM reference signal and detected by the spectrometer. The backscattered excitation light collected by the inner-clad of DCF is finally dissipated into the air at the fuse region between SM fiber and DCF. Although the NA for the core of the DCF is low, the back scattering light is usually strong and the amount of light collected by the core of the DCF is enough for OCM detection. The MPM signal will also be collected by both the core and the inner-clad of the DCF through port 4 (Fig. 2). Only a portion of the signal collected by the clad of the DCF will finally be detected by the photomultiplier tube (PMT) and the MPM signal collected by the core of the DCF will not be sent for detection. By comparing the diameter and NA of the photonic crystal fiber (PCF) core with those of the PCF inner-clad, we determined that the signal collected by the core of the PCF is much less than that collected by the inner-clad of the PCF. It is acceptable to neglect the signal collected by the core of the PCF. The detectable portion of the collected MPM signal is the portion that goes through the multimode fibers [ports 2 and 3 (Fig. 2)]. This portion of the MPM signal is finally detected by the PMT after passing through a bandpass filter. In order to test the efficiency of the device, we launched a red color $(633 \mathrm{~nm}) \mathrm{CW}$ beam from a Helium-Neon laser into the device from port 4 (Fig. 2). The test was performed before the combiner was fused to the SM fiber coupler. The light intensity coming out from ports 1,2 , and 3 (Fig. 2) were measured. It was found that port 2 and port 3, respectively, took $10 \%$ of the total output and port 1 took $80 \%$ of the total output. This shows that with the $(2+1): 1$ PSC and the current setup, $20 \%$ of the light collected by port 4 will go though the two multimode fibers and be detected by the PMT. It should be pointed out that the amount of signal that could be sent for detection in this scheme is dependent on the number of multimode fibers used. The amount of detectable signal can be increased by fusing more multimode fibers with the PCF. With a $(6+1): 1$ and even $(19+1): 1$ pump/signal combiner, detecting more than $50 \%$ of the totally collected power is possible with PSC.

The images shown in Figs. 3(a) and 3(b) are OCM and MPM images of $15 \mu \mathrm{m}$ diameter red fluorescence beads (Invitrogen Corporation, Carlsbad, California) deposited on a cover glass. OCM and MPM images show, respectively, the structural and functional images of the sample. The optical power on the sample is $5 \mathrm{~mW}$. Because a Fourier domain OCM was used here, several en-face depth images could be obtained at once. The OCM image shown in Fig. 3(a) was obtained by summing up all the en-face images at different depths. From the images, we can find that MPM images provide a better contrast than the OCM images. However, the MPM image only shows information of beads and lost the information from other parts. The ability of the system to perform simultaneously second harmonic generation (SHG) imaging and OCM imaging was also tested. The potassium titanyl phosphate (KTP) crystal powder was imaged with the multimodal imaging system. The OCM and SHG images are shown in Figs. 3(c) and 3(d).

We tested the fiber-based combined OCM/MPM system by imaging a biological sample. OCM and MPM are based on different contrast mechanisms and provide different information
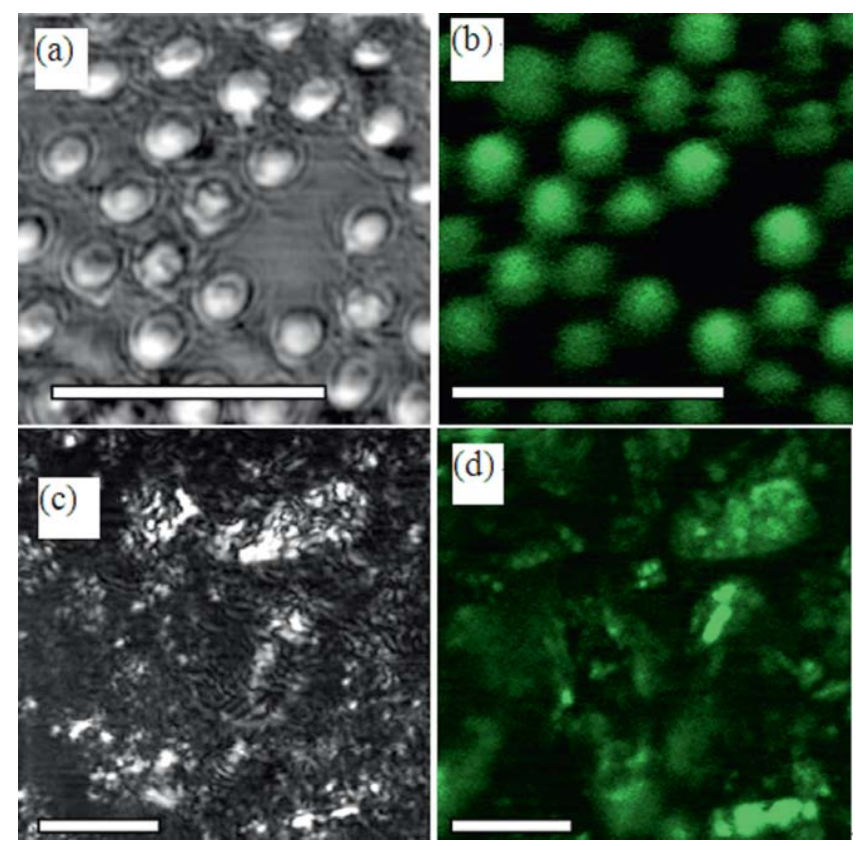

Fig. 3 (a) OCM and (b) TPEF images of red fluorescence beads. (c) OCM and (d) SHG images of KTP crystal powder. Scale bar: $50 \mu \mathrm{m}$.

about the sample. We can find the difference between the OCM and MPM images from a thin slice histology sample in Figs. 4(a) and 4(b). The optical power on the sample side is around $20 \mathrm{~mW}$. The sample is a $6 \mu \mathrm{m}$ thin slice of fixed rabbit heart tissue stained with hematoxylin and eosin. The MPM contrast originates from the two-photon excited fluorescence (TPEF) of the eosin, which is a pinkish-red dye which has a typical one photon absorption peak around $527 \mathrm{~nm} .{ }^{13}$ The MPM image [Fig. 4(b)] shows a clear structure of the thin slice.
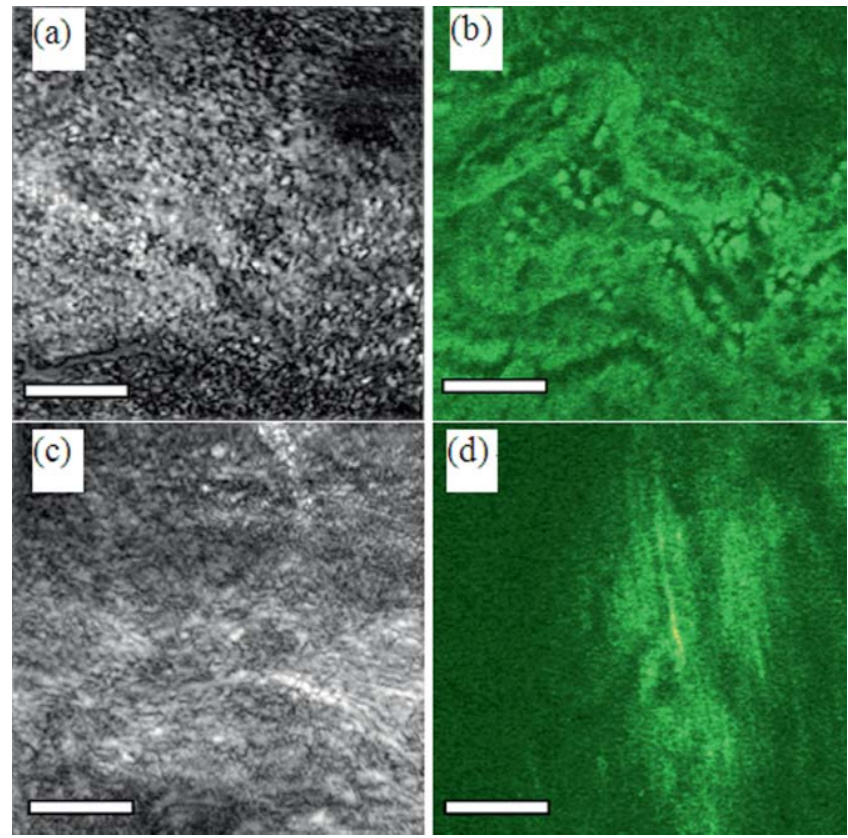

Fig. 4 (a) OCM and (b) SHG images of a thin slice of fixed rabbit heart stained with hematoxylin and eosin. (c) OCM and (d) SHG images of a rat tail tendon. Scale bar: $50 \mu \mathrm{m}$. 
However, the OCM image [Fig. 4(a)] does not show much information about the thin slice, and it shows that the thin slice has a quite uniform scattering property. Figures 4(c) and 4(d) demonstrate the capability of our system to produce simultaneous OCM and MPM images on an unstained rat-tail tendon. The MPM image [Fig. 4(d)] originates its contrast from the SHG signal of the collagen fibrils. The unstained sample usually requires very high light intensity to produce an MPM signal. The optical power on the sample side is around $60 \mathrm{~mW}$ in this experiment, and the required power may be reduced by using a higher numerical aperture objective. The MPM signal was excited only in the central and right part of the sample as shown in Fig. 4(d). This is because the sample surface is not flat and the left part of the sample is defocused. However, defocusing has much less effect on the OCM image [Fig. 4(c)]. The result shows the complimentary nature of OCM and MPM technology.

In conclusion, we have developed a fiber-based multimodal endomicroscopy system that can obtain MPM and OCM images simultaneously. The system used a single fiber-based femtosecond laser as the light source with a central wavelength of around $1.04 \mu \mathrm{m}$. A double-clad device: a pump/signal combiner was proposed to build a compact multimodal system. The double-clad device delivers an excitation beam with its core and collects the signal for OCM and MPM with its core and inner-clad, respectively. With the current setup, the device can confine all the excitation light in the core and has a collection efficiency of $20 \%$ for the MPM signal. The efficiency can be further increased by fusing more multimode fibers with the DCF. Simultaneous second harmonic generation, two-photon excitation fluorescence, and optical coherent microscopy imaging were demonstrated.

\section{Acknowledgments}

This work was supported by the National Institutes of Health (EB-00293, EB-10090, RR-01192, HL-103764, and HL105215), Air Force Office of Scientific Research (FA9550-040101), and the Beckman Laser Institute Endowment.

\section{References}

1. E. Beaurepaire, L. Moreaux, F. Amblard, and J. Mertz, "Combined scanning optical coherence and two-photon-excited fluorescence microscopy," Opt. Lett. 24, 969-971 (1999).

2. C. Vinegoni, T. Ralston, W. Tan, W. Luo, D. L. Marks, and S. A. Bopparta, "Integrated structural and functional optical imaging combining spectral-domain optical coherence and multiphoton microscopy,"Appl. Phys. Lett. 88, 053901 (2006).

3. S. Tang, T. B. Krasieva, Z. Chen, G. Tempea, and B. Tromberg, "Combined multiphoton microscopy and optical coherence tomography using a 12-fs broadband source," J. Biomed. Opt. 11, 020502 (2006).

4. B. W. Graf, Z. Jiang, H. Tu, and S. A. Boppart, "Dual-spectrum laser source based on fiber continuum generation for integrated optical coherence and multiphoton microscopy," J. Biomed. Opt. 14, 034019 (2009).

5. S. Yazdanfar, Y. Y. Chen, P. T. C. So, and L. H. Laiho, "Multifunctional imaging of endogenous contrast by simultaneous nonlinear and optical coherence microscopy of thick tissues," Microsc. Res. Tech. 70, 628633 (2007)

6. H. Bao, S. Y. Ryu, B. H. Lee, W. Tao, and M. Gu, "Nonlinear endomicroscopy using a double-clad fiber coupler," Opt. Lett. 35, 995-997 (2010).

7. G. Liu, K. Kieu, F. W. Wise, and Z. Chen, "Multiphoton microscopy system with a compact fiber-based femtosecond-pulse laser and handheld probe," J. Biophoton. 1-2, 34-39 (2011).

8. M. Balu, T. Baldacchini, J. Carter, T. B. Krasieva, R. Zadoyan, and B. J. Tromberg, "Effect of excitation wavelength on penetration depth in nonlinear optical microscopy of turbid media," J. Biomed. Opt. 14 010508 (2009).

9. M. T. Myaing, J. Y. Ye, T. B. Norris, T. Thomas, J. R. Baker, Jr., W. J. Wadsworth, G. Bouwmans, J. C. Knight, and P. St. J. Russell, "Enhanced two-photon biosensing with double-clad photonic crystal fibers," Opt. Lett. 28, 1224-1226 (2003).

10. D. Bird and M. Gu, "Compact two-photon fluorescence microscope based on a single-mode fiber coupler," Opt. Lett. 27, 1031-1033 (2002).

11. L. Fu and M. Gu, "Double-clad photonic crystal fiber coupler for compact nonlinear optical microscopy imaging," Opt. Lett. 31, 1471-1473 (2006).

12. F. Gonthier, L. Martineau, N. Azami, M. Faucher, F. Séguin, D. Stryckman, and A. Villeneuve, "High-power, All-fiber components: The missing link for high power fiber lasers," Proc. SPIE 5335, 266-276 (2004).

13. H. Du, R. A. Fuh, J. Li, A. Corkan, and J. S. Lindsey, "PhotochemCAD: A computer-aided design and research tool in photochemistry," Photochem. Photobio. 68, 141-142 (1998). 